\title{
TOPOGRAPHY-INDUCED FOCUSING OF RANDOM WAVES
}

\author{
P.B. Smit ${ }^{1}$, T.T. Janssen ${ }^{2}$ and T.H.C.Herbers ${ }^{3}$
}

\begin{abstract}
Refraction of narrow-band surface waves in coastal areas can result in wave-focal zones where due to interference, wave statistics vary rapidly and on similar length scales as those of individual waves. However such interference patterns, or wave coherence, are not accounted for in conventional stochastic wave models that are based on the energy balance equation or radiative transfer equation. In this work we present a quasi-coherent theory, which is an extension of the radiative transfer equation and quasi-homogeneous theory. We show that this new stochastic modelling approach can resolve rapid variations in wave statistics that occur in the vicinity of a wave caustic. The results compare favourably to those obtained from ensemble averages calculated with a deterministic phase resolving model (SWASH) and, in a focal zone, constitute a significant improvement over those obtained with a conventional stochastic wave model based on an energy balance equation (SWAN).
\end{abstract}

Keywords: Wave focusing; Random waves; Stochastic wave model; Diffraction; Wave interference

\section{INTRODUCTION}

The evolution of ocean waves in coastal areas is strongly affected by variations in depth and currents, which has important implications for wave-driven coastal circulation and nearshore transport processes (e.g. Hoefel and Elgar, 2003). To model wave-driven coastal dynamics requires statistical information of the wave field, such as the wave spectrum, variance, and other (integral) parameters (e.g. significant wave height, peak period). For many coastal applications (both scientific and engineering) such wave statistics are obtained from stochastic wave models (e.g. The WAMDI Group, 1988; Tolman 1991; Booij et al. 1999), that are based on some form of the wave action balance (or radiative transfer) equation. These models were originally developed for open ocean application (e.g. The WAMDI Group) and later extended to include shallow-water physics (e.g. depth-induced breaking, triad wave-wave interactions), and are now routinely applied in the coastal zone (Wise Group, 2007).

The radiative transfer equation assumes that the wave field is Gaussian and quasi-homogeneous. These assumptions are often reasonable in the open ocean, however closer to shore, the interaction with two-dimensional topography and currents can result in areas with strongly inhomogeneous statistics (e.g. Magne et al., 2007). In such areas, wave statistics can exhibit fast-scale variations associated with cross-correlations between non-collinear wave components, induced by refraction of a coherent incident wave field; such cross-correlations (the statistical equivalent of a wave interference pattern) are not considered by the radiative transfer equation (e.g. O’Reilly and Guza, 1991). In addition to these linear effects, ocean wave statistics near the shore are often strongly non-Gaussian (e.g. Herbers et al. 2003; Janssen et al. 2006) and involve higher-order correlations (bispectrum). It is thus clear that shallow water effects fundamentally limit the use of the radiative transfer equation in coastal areas.

The difficulty with incorporating such cross-correlations (or higher-order correlations for that matter) is that there are no conservation principles available on the basis of which an evolution equation for these contributions can be derived. Such evolution equations have been derived for special cases (e.g. Agnon \& Sheremet, 1997; Herbers et al., 2003; Janssen et al., 2008; Alber, 1978; Stiassnie et al., 2008), but these approaches require additional assumptions (e.g. one-dimensional medium variations, bandwidth and/or directional restrictions) and are thus of limited use for operational wave models.

In the present work we discuss results from a new stochastic modeling approach (see Smit \& Janssen, 2012), which is a consistent extension of the radiative transfer equation to incorporate wave inhomogeneity induced by refraction over two-dimensional topography. The model is derived from general principles and is valid for waves of arbitrary bandwidth propagating through a variable medium. In this approach the wave statistics are described by a Coupled-Mode (or Wigner-Ville) spectrum, which accounts for the coherent interference between wave components, and resolves rapid variations in wave statistics associated with such wave interferences.

The model is based on what we refer to as a Quasi-Coherent (QC) theory, which involves a transport equation for the Coupled-Mode (CM) spectrum. We will discuss how this new approach

\footnotetext{
${ }^{1}$ Faculty of Civil Engineering and Geosciences, Delft University of Technology, Stevinweg 1, Delft, 2628CN, The Netherlands.

${ }^{2}$ Theiss Research, 7411 Eads Avenue, La Jolla, CA 92037, USA

${ }^{3}$ Department of Oceanography, Naval Postgraduate School, 1 University Circle, Monterey, CA 93943, USA
} 
relates to the radiative transfer equation by means of theory and an example of topography-induced focusing of a narrow-banded wave field propagating over a circular shoal. In our discussion we compare results of the new QC model to a conventional stochastic model based on the radiative transfer equation (SWAN, Booij et al., 1999), and statistical averages computed through Monte-Carlo simulation with a deterministic, phase-resolving model (SWASH, Zijlema et al., 2011).

Our discussion here is limited to inhomogeneous wave fields and the linear effects associated with refractive focusing of coherent random wave fields. However, the same reasoning and theoretical framework can be applied to non-Gaussian effects and the evolution of higher-order correlations.

\section{A QUASI-COHERENT MODEL IN SHALLOW WATER}

To account for wave interference in random wave fields propagating over variable topography, we consider an approximate transport equation for the second-order statistics of the free surface elevation $\eta(\mathbf{x}, t)$ (assumed to be a zero-mean random wave variable), as a function of the horizontal coordinates $\boldsymbol{x}=(x, y)$ and time $t$, which can be written as (Smit \& Janssen, 2012)

$$
\partial_{t} E+\mathbf{c}_{\mathbf{x}} \cdot \nabla_{\mathbf{x}} E+\mathbf{c}_{k} \cdot \nabla_{\mathbf{k}} E=2 \operatorname{Re}\{S\}
$$

where $E(\mathbf{k}, \mathbf{x}, t)$ is referred to here as a coupled-mode spectrum, $\mathbf{c}_{\mathbf{x}}$ is the transport velocity through geographical space, and $\mathbf{c}_{k}$ the transport velocity through spectral space. The LHS is of similar form as the well-known radiative transport equation, and the 'source' term on the RHS accounts for wave coherence; the latter has the general form

$$
S(\mathbf{k}, \mathbf{x})=-i \int K\left(\mathbf{k}, \mathbf{q}, \mathbf{x}, i \nabla_{\mathbf{x}}\right)\left[E\left(\mathbf{k}-\frac{\mathbf{q}}{2}, \mathbf{x}, t\right)-E(\mathbf{k}, \mathbf{x}, t)+\frac{1}{2} \mathbf{q} \cdot \nabla_{\mathbf{k}} E(\mathbf{k}, \mathbf{x}, t)\right] d \mathbf{q},
$$

where $K$ is an integration kernel (to be defined later) and $\mathbf{q}$ is a difference wavenumber. The presentation in equation (1.1), in which effects associated with coherence are written as a source term, is somewhat arbitrary, and chosen here to make the resemblance and differences with the radiative transfer equation explicit. The equation (1.1) is what we refer to as the Quasi-Coherent $(\mathrm{QH})$ approximation and it differs from the radiative transfer equation (or Quasi-Homogeneous approximation) through the presence of the source term S (and thus the difference between $\mathrm{E}$ and a variance density function).

However, equation (1.1) should not generally be interpreted as a radiative transfer model with a source term. In particular, the spectrum $E$ accounts both for variance and potentially cross-variance contributions and is defined as the Fourier transform of a two-point spatial correlation function with respect to the separation distance, which can be written as

$$
E(\mathbf{k}, \mathbf{x}, t)=\frac{1}{(2 \pi)^{2}} \int\langle\eta(\mathbf{x}+\xi / 2, t) \eta(\mathbf{x}-\xi / 2, t)\rangle \exp (-i \mathbf{k} \cdot \xi) d \xi
$$

Here $\xi$ is the separation distance and the angle brackets denote the ensemble average. As a consequence, the spectrum $E$, although real, is not always positive, and cannot generally be interpreted as an energy (or variance) density spectrum. To make this distinction explicit, we refer to $E$ as a coupled-mode (CM) spectrum. The definition of $E$ in equation (1.2) implies that it describes the complete linear statistics of a random wave field $\eta$, including interference (or coupling) between different spectral constituents associated with rapid variations in the statistics. Note that, although $E$ is not a variance density, the local wave variance can still be obtained from the spectral integral

$$
V(\mathbf{x}, t)=\int E(\mathbf{k}, \mathbf{x}, t) d \mathbf{k}
$$

Of course, if the wave statistics are quasi-homogenous the CM-spectrum reduces to a variance density spectrum, the source term $S$ in (1) becomes negligible, and equation (1) reduces to the usual radiative transfer equation. Hence, equation (1) contains Quasi-Homogeneous theory (the radiative transfer 
equation) as a special case, but remains valid in wave interference zones where the statistics undergo potentially rapid (on intra-wave scales) variations.

In this work we consider steady solutions to equation (1), where coherent wave interference is generated through interaction of a narrow-band coherent wave field with two-dimensional medium variations. Further, for convenience, we will assume that waves are forward-scattering so that we can solve equation (1) using a forward marching finite-difference algorithm in the x-direction. Along each gridline $x_{m}\left(x_{m}=x_{0}+m \Delta x, \quad m=0 \ldots M\right.$, with $\Delta x$ a constant mesh-size $)$ a first-guess solution is obtained from the steady-state solution of the LHS of equation (1), using second-order spatial and spectral upwind approximations. Subsequently, the approximate solution is marched forward in time, including a quadrature approximation to equation (2), using a $4^{\text {th }}$-order Runge-Kutta method until a steady state is reached. Although our numerical implementation for this paper assumes forward-scattering of the waves, equation (1) is fully isotropic and has no fundamental restrictions on wave propagation direction. For more general wave evolution, our numerical implementation can be easily extended to include waves traveling in the full plane by applying the same algorithm iteratively in two or four separate sweeps, similar to the method employed in the SWAN model (Booij et al., 1999).

\section{WAVE FOCUSING BEHIND A SHOAL IN SHALLOW WATER}

To illustrate the difference between the Quasi-Coherent theory and Quasi-Homogeneous theory, and demonstrate that the QC stochastic theory accurately resolves coherent interference patterns in focal regions, we consider the evolution of wave statistics over a circular shoal on an otherwise (approximately) flat bed. To verify model predictions, we compare results to those obtained by averaging results of a deterministic, shallow-water, time domain model (SWASH, Zijlema et al., 2011), and contrast the results with those obtained from a radiative transport model (SWAN, Booij et al.,1999).

For this example we consider shallow-water waves so that $c_{\mathbf{x}}=\mathbf{k} c / k$ and , $c_{k}=-\nabla_{\mathbf{x}} c$ where $c(\mathbf{x})$ denotes the shallow water wave celerity $(=\sqrt{g h})$ and $k(=|\mathbf{k}|)$ is the wavenumber magnitude. In shallow water the integration kernel $K$ in equation (2) can be written in explicit form as

$$
K\left(\mathbf{k}, \mathbf{q}, \mathbf{x}, i \nabla_{\mathbf{x}}\right)=\hat{c}(\mathbf{q}) \exp (i \mathbf{q} \cdot \mathbf{x})\left(|\mathbf{k}|-i \frac{1}{2} \frac{\mathbf{k}}{|\mathbf{k}|} \cdot \nabla_{\mathbf{x}}\right)
$$

The restriction to shallow water here is a convenient choice and simplifies the numerical implementation, but an extension to arbitrary depth is straightforward and merely requires substitution of a more general form of the kernel function $\mathrm{K}$.

Further, we chose a topography such that the shallow water wave celerity is given as a Gaussian shaped disturbance to an otherwise constant value $c_{\infty}$ so that wave celerity $c(\mathbf{x})$, and its Fourier transform $\hat{c}(\mathbf{k})$ that is used in equation (5), are of the form

$$
c(\mathbf{x})=c_{\infty}-\Delta c \exp \left[-\frac{\mathbf{x} \cdot \mathbf{x}}{L^{2}}\right] \quad \text { and } \quad c(\mathbf{k})=c_{\infty} \delta(\mathbf{k})-\frac{\Delta c}{4 \alpha \pi} \exp \left[-L^{2} \frac{\mathbf{k} \cdot \mathbf{k}}{4}\right] .
$$

Here $c_{\infty}$ is the wave celerity in the far field (as $\mathbf{x} \rightarrow \pm \infty$ ) and $\Delta c$ represents the height of the disturbance at the origin which is set to $\Delta c=0.25 c_{\infty}$ (so that $h_{0}=9 / 16 h_{\infty}$, with $h_{0}$ representing the depth over the shoal). Furthermore the topography length scale is $L=4 L_{p}$, where $L_{p}$ corresponds to the far field wavelength at the peak of the incident spectrum.

The incident wave-field is characterized by its (homogeneous) far field spectrum $\mathcal{E}_{\infty}(f, \theta)$, represented here as

$$
E_{\infty}(f, \theta)=D(\theta) \frac{m_{0}}{\sigma_{f} \sqrt{2 \pi}} \exp \left[-\frac{\left(f-f_{p}\right)^{2}}{2 \sigma_{f}^{2}}\right] \text { with } m_{0}=\iint E(f, \theta) d f d \theta .
$$

where $f$ and $\theta$ are frequency and direction respectively, $f_{p}$ is the peak frequency, $\sigma_{f}=0.1 f_{p}$, and $m_{0}$ represents the total variance. The waves are normally incident (mean direction along $\mathrm{x}$ ) and the 
directional distribution $D(\theta)$ is represented by a raised-cosine $\cos ^{m}\left(\theta-\theta_{0}\right)$ with $\mathrm{m}=800$ (corresponding to $2^{\circ}$ directional spreading, Kuik et al., 1988). To ensure shallow-water conditions we choose $k_{p} h_{\infty}=0.01$, where $k_{p}$ is the wavenumber associated with the peak frequency $f_{p}=0.5 \pi^{-1} k_{p} \sqrt{g h_{\infty}}$ and $h_{\infty}$ is the depth in the far field (as $\mathbf{x} \rightarrow \pm \infty$ ). In addition, to ensure that the non-linear effects in the deterministic model are negligible, we make the wave amplitude arbitrarily small and set $k_{p} H_{m 0}^{\infty}=H_{m 0}^{\infty} / h_{\infty}=10^{-4}$, where $H_{m 0}^{\infty}=4 \sqrt{m_{0}}$.

(a)

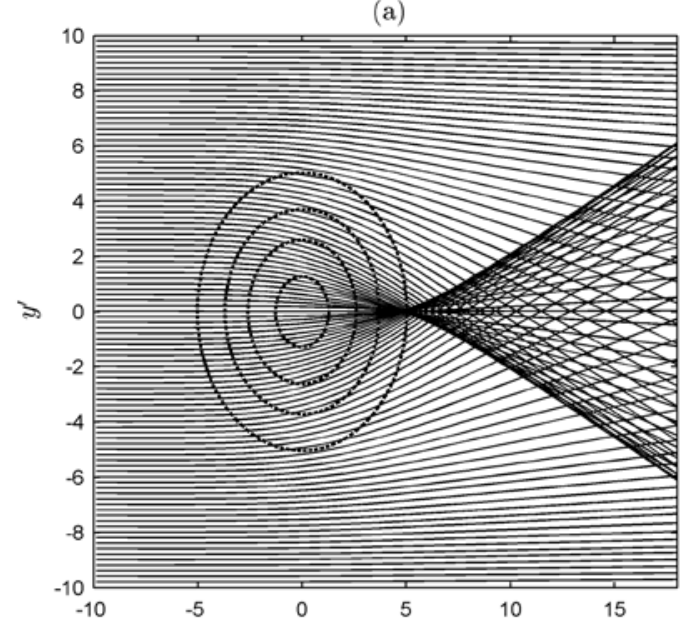

(c)

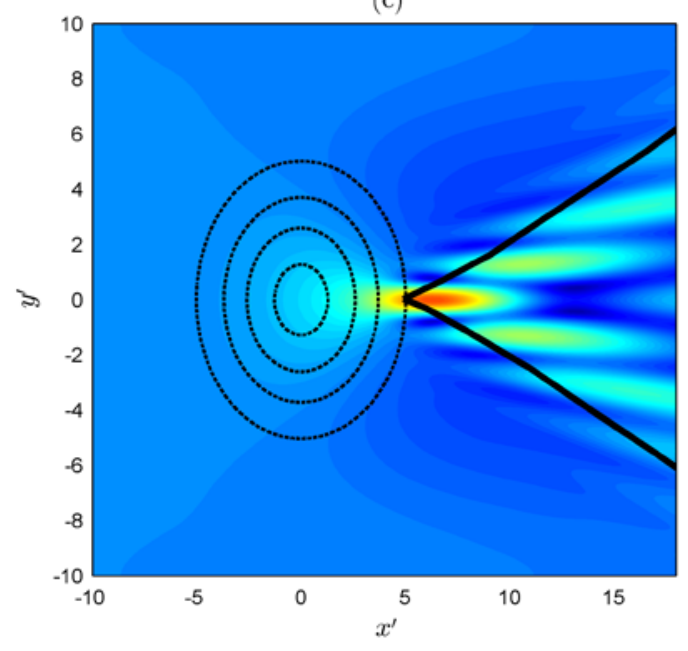

(b)

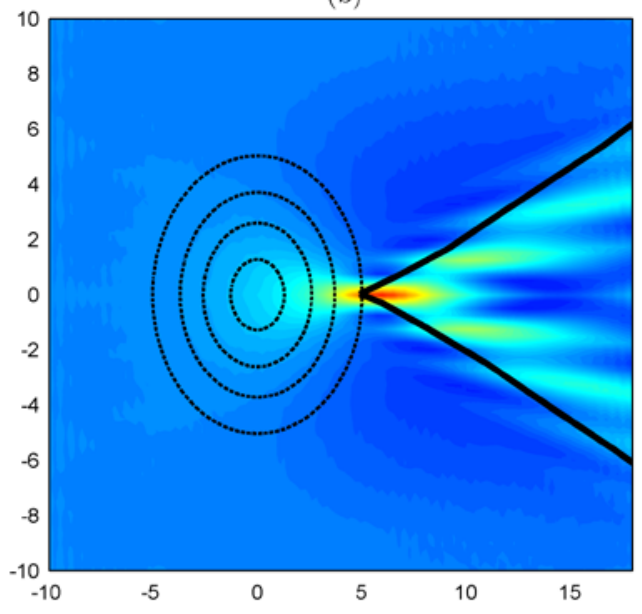

(d)

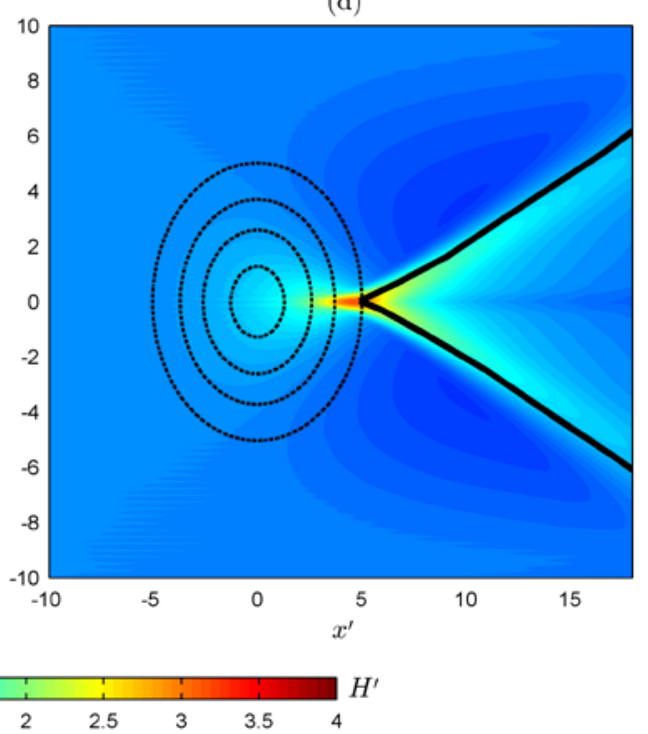

Figure 1. Evolution of a narrow band wave field over a circular shoal. Shown are wave rays for the peak frequency (panel a), and normalized wave heights $H^{\prime}$ (normalized by the incident far field value) computed by SWASH (panel b), the present model (panel c), and the radiative transfer equation (SWAN, panel d). Dashed lines indicate depth contours (drawn between $0.6 h_{\infty}$ and $0.9 h_{\infty}$ at equidistant intervals of $0.1 h_{\infty}$ ) solid thick lines indicate (approximately) location of caustics. The $\left(x^{\prime}, y^{\prime}\right)$ are the horizontal coordinates normalized with the incident peak wavelength.

We consider wave evolution in a rectangular domain of $18 \times 20$ wavelengths (such that $-10 L_{p} \leq x \leq 18 L_{p}$ and $-10 L_{p} \leq y \leq 10 L_{p}$ ), uniformly discretized with $\Delta x=\Delta y=L_{p} / 8$ for the QC and SWAN simulations, and $\Delta x=\Delta y=L_{p} / 80$ for the SWASH model. The wavenumber domain in the QC model is uniformly discretized with $\Delta k_{x}=\Delta k_{y}=k_{p} / 80$ over the range $k_{p} / 2 \leq k_{x} \leq 2 k_{p}$, and $-3 k_{p} / 2 \leq k_{y} \leq 3 k_{p} / 2$. In SWAN, the directional domain is limited to $-50^{\circ} \leq \theta \leq 50^{\circ}$ (with $\Delta \theta=0.1^{\circ}$ ) and frequency domain ranges $0.7 f_{p} \leq f \leq 1.3 f_{p}$ (discretized using 20 logarithmically distributed frequencies). To obtain a reliable estimate of the variance in SWASH, the simulation duration was 400 peak periods ( $\Delta t \approx T_{p} / 126$ ) of which the first hundred periods were discarded. 
At the incident wave boundary ( $x=-10 L_{p}$ ) the far field spectrum (7) is imposed on the QC and SWAN model. The same boundary condition is used for SWASH to obtain realizations of particle velocities using random phases and linear wave theory. In SWAN and the QC model a radiative condition was used on the remaining boundaries. To minimize reflection in SWASH, a sponge layer (two wavelengths deep) was combined with a weakly reflective condition along the boundary at $x=20 L_{p}$. No additional measures were imposed along the lateral boundaries in SWASH which therefore were fully reflective. To provide a meaningful comparison, and illustrate the differences between the present QC approximation and Quasi-Homogeneous theory, SWAN was run as a numerical implementation of the classical radiative transport without any source terms, and without the phase-averaged diffraction approximation (Holthuijsen et al., 2003).

(a) $x^{\prime}=6$

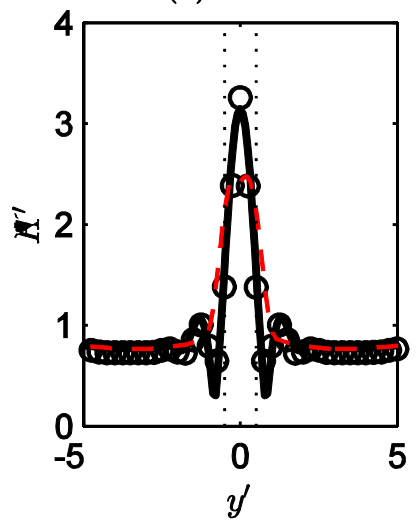

(d) $y^{\prime}=0$

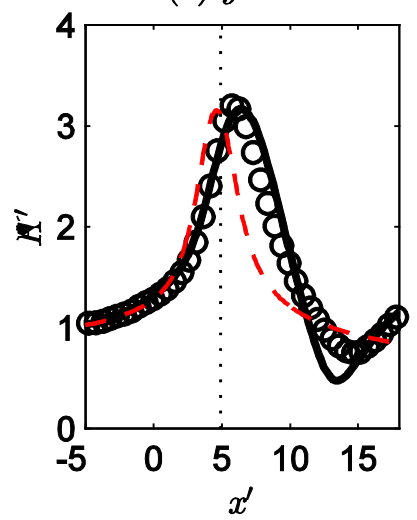

(b) $x^{\prime}=10$

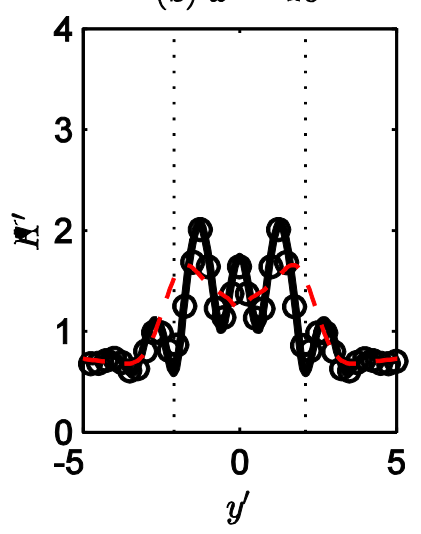

(e) $y^{\prime}=2$

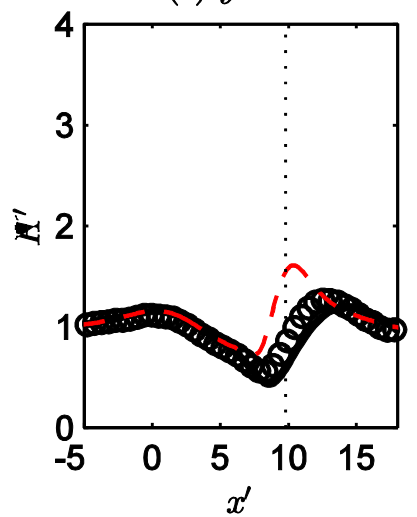

(c) $x^{\prime}=14$

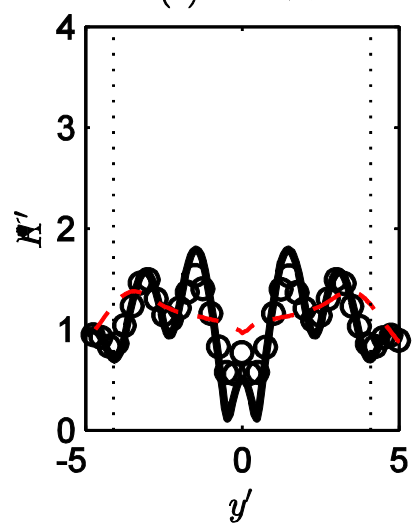

(f) $y^{\prime}=4$

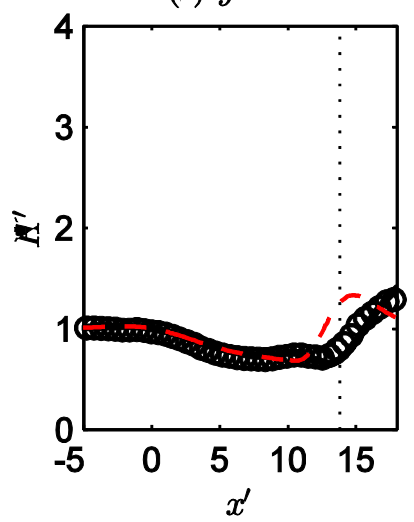

Figure 2. Shown are waves heights $H^{\prime}$ (normalized by the incident far field value) computed by SWASH (circles), present model (solid line), and SWAN (dashed red line) along lateral transects (panel a-c) and longitudinal transects (panel $d$-f). The vertical dotted lines indicate an approximate crossing between a transect and a caustic line (as shown in figure 1). The $\left(x^{\prime}, y^{\prime}\right)$ are the horizontal coordinates normalized with the incident peak wavelength.

A wave ray analysis (geometric optics) of wave propagation over the shoal (see Fig. 1a) shows the convergence of rays in a focal point behind the shoal, from where two caustics develop (Fig. 1a). In the area enclosed by these caustics, we anticipate coherent interference of waves and thus rapid variations in wave statistics. This is confirmed in the results from the deterministic model (Fig. 1b), which shows fast lateral variations in wave height, with the largest wave energy occurring just behind the focal point, consistent with the ray-traced solution. The fine-scale structure of the wave energy in this area is accurately reproduced by the QC model (Fig. 1c). In contrast, the result predicted by the radiative transfer equation (SWAN) is quite different, and does not resolve the fine-scale structure that is associated with wave coherency and the refraction-induced interference patterns.

Inspection of different lateral transects behind and longitudinal transacts across the shoal (Fig. 2) further confirm (more quantitatively) that the QC-approximation accurately resolves the interference pattern. In the lateral cross-sections behind the focal point (Fig. 2b-c), the fast-scale lateral oscillatory 
pattern is represented by the QC-approximation, albeit with slight over- and under-predictions of the respective minima and maxima (e.g. Fig. 2c). Furthermore the maximum wave height, found in the focal point just behind the crest of the shoal, is correctly reproduced (Fig. 2d).

In general, outside the area enclosed by the caustics (where no wave rays cross), the geometric optics approximation implied by the radiative transport equation is - as expected - a very good approximation. In the vicinity of the caustics however, and in the focal region enclosed by the caustics, the inclusion of cross-variance contributions, as present in the QC model, is essential to accurately resolve the rapid variations in wave statistics. This is likely to be important in natural focal zones in coastal areas where waves are refractively focused for instance over tidal currents in inlets, or coastal bathymetry.
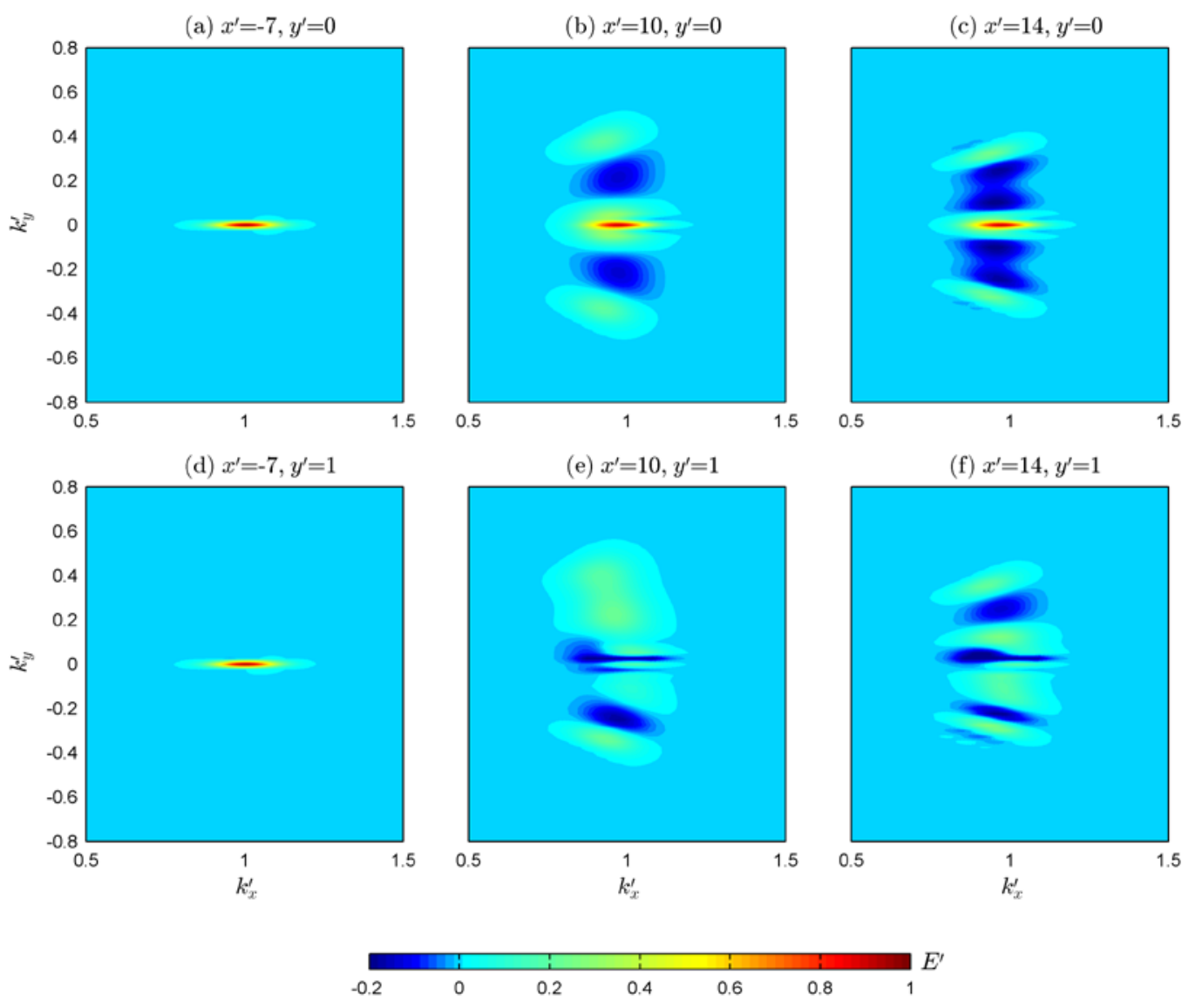

Figure 3. Normalized Coupled-mode (CM) spectraError! Objects cannot be created from editing field codes. (normalized with the peak of the incident spectrum) at six locations in the domain. Top panels: CM spectra at three locations alongError! Objects cannot be created from editing field codes.; bottom panels: CM spectra at three locations along lineError! Objects cannot be created from editing field codes.. In the region of wave inhomogeneity a relatively small spatial offset results in large changes in the CM spectrum due to rapid changes in the cross-variance contributions. Changes in the auto-variance contributions are small on these spatial scales. The (Error! Objects cannot be created from editing field codes.) are the wavenumber coordinates normalized with the incident peak wavenumber.

These cross-variance contributions appear in the CM spectrum as additional peaks in the spectrum, located midway (spatially and spectrally) between the auto-variance contributions of the correlated wave components (Hlawatsch \& Flandrin, 1997), and oscillate on the scale of the interference pattern. To substantiate this, we can consider the variance density spectrum (as predicted by QH theory) as it evolves along the central wave ray $(y=0)$. Prior to the focal point, the spectrum contains a single peak related to a slightly deformed version of the incident spectrum. Past the focal point we anticipate that, due to the crossing wave rays, three peaks emerge: one that propagates along the central ray, and two contributions at opposite, but equal angles with the mean direction. 
The evolution of the CM spectrum is different. Before the shoal, where the CM spectrum is essentially a variance density spectrum. it starts as a single concentrated contribution (Fig. 3a) which then evolves into three positive auto-variance peaks augmented by additional cross-variance contributions, each located midway between auto-variance contributions associated with the correlated wave components (Fig. 3b-c). For instance, at $x^{\prime}=10$ (Fig. 3b), these cross-contributions manifest themselves as two negative peaks at $k_{y}^{\prime} \approx \pm 0.2$ (with $k_{y}^{\prime}=k_{y} / k_{p}$ ), located between the auto-variance contributions at $k_{y}^{\prime}=0$ and $k_{y}^{\prime} \approx \pm 0.4$; and a third contribution at $k_{y}^{\prime}=0$ that carries the information regarding the correlation between the outer auto-variance peaks at $k_{y}^{\prime} \approx \pm 0.4$. Consequently, the central peak at $k_{y}^{\prime}=0$ consists of an auto-variance contribution, with a cross-variance contribution superimposed.

When the spectrum is evaluated at a slightly offset location $\left(y^{\prime}=1\right)$ the spectrum changes rapidly in the region enclosed by the caustics (Fig. 3e-f), whereas only small lateral variations are observed before the shoal (Fig. 3d). In part, the observed variation can be attributed to changes in the local wave-ray configuration, since each auto-variance contribution now propagates in a slightly modified direction. However, variations on auto-variance contributions are slow and the observed variation is primarily due to rapid changes in the cross-variance contributions, which vary on wavelength scales as they measure the statistical phase relation between two wave components. In other words, the cross-variance contributions change from positive when two components interfere constructively, to negative when two components interfere destructively and their values thus oscillate on the scale of the interference pattern, resulting in the observed rapid changes in the CM spectrum within the region enclosed by the caustics.

\section{DISCUSSION}

A primary difference between Quasi-Homogeneous $(\mathrm{QH})$ theory and the present Quasi-Coherent (QC) theory lies in the definition of the second-order wave statistical variable $E$. Whereas in QH theory the spectrum is directly interpretable as a spectral variance (or energy) distribution function, in QC theory this is no longer true. Instead, in addition to auto-variance contributions, the CM spectrum contains cross-variance contributions, which carry information about the correlation between non-collinear waves. Since such cross-variance contributions do not directly correspond to a particular wave field component, they do not carry energy; instead these components carry additional (cross-phase) information regarding the correlation of two wave components. In terms of the wave energetics, these terms dictate how the energy of the wave field is distributed between kinetic and potential energy. In a statistically homogeneous wave field, wave energy is equipartitioned so that knowledge of the potential energy (the auto-variance contributions) uniquely determines the energy content of the wave field. In an inhomogeneous wave field this is no longer true, and information on the distribution of potential and kinetic energy is required to fully characterize the wave field statistics. This information is not available in the variance density spectrum, but in the CM spectrum this is carried by the cross-variance contributions.

As can be seen from the refraction example considered here (Fig 1-2) QC theory incorporates effects commonly associated with wave diffraction. Not unlike the Huygens-Fresnel principle (e.g. Born and Wolf, 1999), in QC theory diffraction effects are captured by accounting for the coherency of the interference phenomenon, by carrying cross-phase information in the wave evolution. The theory is an approximation in the sense that it accurately resolves coherence between components up to intermediate mutual angles (up to angles of $30^{\circ}$, Smit and Janssen, 2012). Despite this theoretical limitation, the model can accurately reproduce classic diffraction patterns associated with wide-angle diffraction behind a semi-infinite breakwater or a breakwater gap (Smit and Janssen, 2011) and in practice the model appears well suited for larger-angle cross-correlations also. .

The QC theory is therefore a consistent extension of QH theory and as such provides a framework for the extension of models based on the radiative transfer equation to account for cross-correlations in the wave field. Arguably, such cross-correlations can alternatively be resolved with deterministic phase-resolving models. However, such models require high grid resolutions everywhere (to resolve individual waves) whereas QC theory contains QH theory as a special case and contains fast variations only where the wave statistics vary rapidly (which is usually only the case in localized focal zones), which can be exploited when implemented on an unstructured grid. Fundamentally then, QC theory is a 
new, cross-phase resolving modeling approach in contrast to phase-resolving models, which resolve all the phases of individual waves, and the radiative transfer equation, which resolves none. By extending traditional stochastic modeling paradigms to include cross-phase information (rather than resolving all the individual wave phases) has the advantage that it can readily be coupled to, or incorporated in, existing operational models, and that source terms accounting for the generation (e.g. due to wind), dissipation (e.g. white-capping, depth-induced breaking), and non-linear interactions (e.g. Quadruplets) developed for these models can be readily included.

The theoretical framework developed here can be used also to derive evolution equations for higher-order correlations to study the development and evolution of non-Gaussian statistics in shallow water. This will be discussed elsewhere.

\section{CONCLUSIONS}

In this paper we consider a new Quasi-Coherent stochastic wave theory that includes the effects of coherent interferences in random wave fields, either propagated in from the boundaries or generated through the interaction with medium variations. The theory is fully isotropic and valid for wave fields of arbitrary bandwidth in any water depth. We have presented an example for non-dispersive waves focused over a circular shoal and, through comparison to Monte-Carlo simulations with a deterministic model, shown that Quasi-Coherent theory effectively models wave interference patterns and rapid variations in wave statistics associated with such effects.

\section{ACKNOWLEDGMENTS}

This research is supported by the U.S. Office of Naval Research (Coastal Geosciences Program and Physical Oceanography Program) and by the National Oceanographic Partnership Program. The authors thank Leo Holthuijsen, whose continuing support made it possible for PBS to pursue this research, and Nico Booij, who kindly allowed us to use his ray-tracing software.

\section{REFERENCES}

Agnon, Y. and Sheremet, A. 1997. Stochastic nonlinear shoaling of directional spectra. J. Fluid Mech. 345, 79-99.

Alber, I. E. 1978. The effects of randomness on the stability of two-dimensional surface wavetrains. Proc. R. Soc. London. 363, 525-546.

Booij, N., Ris, R. C. and Holthuijsen, L. H. 1999. A third-generation wave model for coastal regions 1. model description and validation. J. Geophys. Res. 104, 7649-7666.

Born, M. and Wolf, E. 1999. Principles of Optics. Cambridge, Cambridge univ. press, 988 pp.

Herbers, T. H. C. \& Burton, M. C. 1997. Nonlinear shoaling of directionally spread waves on a beach. J. Geophys. Res. 102, 21,101-21,114.

Herbers, T. H. C., Orzech, Mark, Elgar, Steve and Guza, R. T. 2003. Shoaling transformation of wave frequency-directional spectra. J. Geophys. Res. 108, 3013.

Hlawatsch, F. and Flandrin, P. 1997. The interference structure of the Wigner distribution and related time-frequency signal representations., pp. 59-133. Amsterdam, Elsevier.

Hoefel, F. \& Elgar, S. 2003. Wave-induced sediment transport and sandbar migration. Science 299, 1885-1887.

Holthuijsen L.H., Herman A. and Booij N., 2003. Phase-decoupled refraction-diffraction for spectral wave models, Coastal Engineering, 49, 291-305

Janssen, A. and Claasen, T. 1985 On positivity of time-frequency distributions. Acoustics, Speech and Sig. Proc., IEEE Trans. 33 (4), 1029-1032.

Janssen, T. T., Herbers, T. H. C. and Battjes, J. A. 2008. Evolution of ocean wave statistics in shallow water: Refraction and diffraction over seafloor topography. J. Geophys. Res. 113, C03024.

Komen, G.J., Cavaleri, L., Donelan, M., Hasselmann, K., Hasselmann, S. and Janssen, P.A.E.M. 1994 Dynamics and Modelling of Ocean Waves. Cambridge, Cambridge University Press.

Kuik A.J., van Vledder, G.P., and Holthuijsen, L.H. 1988. A method for the routine analysis of pitchand-roll buoy wave data. J. Phys. Ocean., 18,1020-1034

Magne, R., Belibassakis, K. A., Herbers, T. H. C., Ardhuin, F., O’Reilly, W. C. and Rey, V. 2007. Evolution of surface gravity waves over a submarine canyon. J. Geophys. Res. 112, C01002. 
Mandel, L. \& Wolf, E. 1995 Optical coherence and Quantum Optics. Cambridge, Cambridge Univ. Press. 595 pp.

Mase, H. 2001, Multi-Directional Random Wave Transformation Model Based On Energy Balance Equation, Coastal Engineering, 43, 317-337

McWilliams, J. C. \& Restrepo, J. M. 1999 The wave-driven ocean circulation. Oceanogr. 29, 25232540.

O’Reilly, W. C. \& Guza, R. T. 1991 Comparison of spectral refraction and refraction-diffraction wave models. J. Wat , Port Coast. Ocean Eng. 117, 199-215.

Resio, D.T., 1988. A steady-state wave model for coastal applications. Proceedings of $21^{\text {st }}$ Conference on Coastal Engineering, ASCE, 929 - 940.

Penney, W. G. \& Price, A. T. 1952 Part i. the diffraction theory of sea waves and the shelter afforded by breakwaters. Proc. R. Soc. London 244 (882), 236-253.

Smit, P.B. \& Janssen, T.T. 2011, Coherent interference and diffraction in random waves, presented at the 12th International Workshop on Wave Hindcasting and Forecasting. Kohala Coast, Hawaii

Smit, P.B. \& Janssen, T.T. 2012 Coherent structures in random waves. Manuscript in preparation for J. Phys. Ocean.

Stamnes, J.J. 1986 Waves in focal regions: propagation, diffraction, and focusing of light, sound, and water waves. Boston, A. Hilger.

Stiassnie, M., Regev, A. \& Agnon, Y. 2008 Recurrent solutions of alber's equation for random water wavefields. J. Fluid Mech. 598, 245-266.

The WAMDI Group 1988 The wam model - a third generation ocean wave prediction model. J. Phys. Ocean. 18, 1775-1810.

Thomson, J., Elgar, S., Herbers, T. H. C., Raubenheimer, B. \& Guza, R. T. 2007 Refraction and reflection of infragravity waves near submarine canyons. J. Geophys. Res. 112, C10009

Tolman, H. L. 1991 A third-generation model for wind waves on slowly varying, unsteady, and inhomogeneous depths and currents. J. Phys. Ocean. 21, 782-797.

Wise Group 2007 Wave modelling - the state of the art. Progr. In Oceanogr. 75, 603-674.

Zijlema M., Stelling G., and Smit. P.B., 2011, SWASH: An operational public domain code for simulating wave fields and rapidly varied flows in coastal waters. Coastal Engineering, 58, 9921012. 